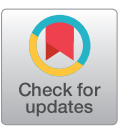

\section{Original Article}

$J$ Cerebrovasc Endovasc Neurosurg. 2020 June;22(2):53-64

Received: 7 January 2020

Revised: 6 February 2020

Accepted: 10 February 2020

\section{Correspondence to Cheol Wan Park}

Department of Neurosurgery, Gachon University Gil Medical Center,

21, Namdong-daero 774beon-gil,

Namdong-gu, Incheon 21565, Korea

Tel +82-32-460-3304

Fax +82-32-460-3899

E-mail cwpark@gilhospital.com

ORCID http://orcid.org/0000-0002-4682-9100

\title{
Risk factors of postoperative remote intracerebral hemorrhage after craniotomy for ruptured cerebral aneurysms
}

\author{
Insu Lee ${ }^{1}$, Cheol Wan Park ${ }^{1,2}$, Chan Jong You ${ }^{1,2}$, Dae Han Choi ${ }^{1,2}$, \\ Kwangwoo Park ${ }^{1,2}$, Young Bo Kim', Woo Kyung Kim', Gi-Taek Yee', \\ Myeong-Jin Kim ', Eun Young Kim ${ }^{1}$ \\ ${ }^{7}$ Department of Neurosurgery, Gil Medical Center, Gachon University, Incheon, Korea \\ ${ }^{2}$ Department of Emergency Medicine, Section of Critical Care Medicine, Gil Medical Center, \\ Gachon University, Incheon, Korea
}

Objective: We aimed to identify the relation between perioperative cerebrospinal fluid (CSF) drain through lumbar drainage (LD) and development of postoperative (POP) remote intracerebral hemorrhage $(\mathrm{rICH})$ in craniotomy to treat ruptured intracranial aneurysms.

Methods: We retrospectively reviewed consecutive patients who underwent craniotomy for ruptured cerebral aneurysms at the authors' institution between 1998 and 2004. We subsequently compared the incidence and characteristics of POP rICH between the patients who had a perioperative LD and those who did not. All statistical analyses were conducted using the software package SPSS 19.0 (SPSS Inc., Chicago, IL, USA). A $p$ value of $<0.05$ was considered statistically significant.

Results: We enrolled 688 patients, of which 80 patients (11.6\%) received perioperative LD, and 608 did not. LD and non-LD groups were comparable because although clinical characteristics of the two groups were significantly different considering history of hypertension, timing of surgery, and closed system negative pressure suction drain (SD) placement, none of these three variables was an independent risk factor associated with $\mathrm{POP} \mathrm{rlCH}$ in multivariate analysis. $\mathrm{POP} r \mathrm{CH}$ incidence was significantly higher in the LD goup (12.5\%) than non-LD group $(0.8 \%)(p=0.000)$ in univariate analysis. LD placement was the only independent risk factor associated with the development of $\mathrm{rICH}$ in multivariate logistic regression analysis.

Conclusions: POP rICH incidence was significantly higher in patients who were managed with perioperative LD than in those who did not. LD insertion in craniotomy for ruptured intracranial aneurysm, should be closely monitored to address the occurrence of POP rICH.

Keywords Intracranial aneurysm, Postoperative hemorrhage, Cerebrospinal fluid, Cerebrospinal fluid leak
This is an Open Access article distributed under the terms of the Creative Commons Attribution Non-Commercial License (http://creativecommons.org/licenses/ by-nc/3.0/) which permits unrestricted noncommercial use, distribution, and reproduction in any medium, provided the original work is properly cited. 


\section{INTRODUCTION}

Draining the cerebrospinal fluid (CSF) via lumbar drainage (LD) is frequently performed in numerous neurosurgical settings and to clip the ruptured intracranial aneurysm for primarily obtaining appropriate brain relaxation, following which minimizing brain retraction, avoiding bloody CSF from obscuring the operative field. However, many adverse events from CSF drain have been established and reported. These include the Arnold-Chiari syndrome, ${ }^{133) 24}$ intracerebral hemorrhage $(\mathrm{ICH}),{ }^{426)}$ intracranial hypotension, ${ }^{2428)}$ local and central nervous system infections, ${ }^{3)}$ peripheral nerve injury, ${ }^{3)}$ part of posterior reversible encephalopathy syndrome (PRES), ${ }^{1732)}$ hydrocephalus (after subarachnoid hemorrhage $[\mathrm{SAH}]),{ }^{14)}$ intraspinal epidermoid tumor, ${ }^{23)}$ brain swelling, ${ }^{1132(34) 37)}$ transtentorial brain herniation, ${ }^{24)}$ and sudden death. ${ }^{11) 32)}$

Postoperative (POP) ICH occurrence is one of the most serious complications in almost all neurosurgical procedures. Remote cerebellar hemorrhage was first introduced in 1977 by Yasargil and Yonekawa ${ }^{36)}$ following a revascularization surgery. Additionally among the several types of POP ICH, a remote ICH (rICH) that was responsible for complicating the cerebral aneurysm surgery, was first reported in 1983 by Waga et al. ${ }^{35}$ Following which, a number of such cases have been published by different authors. ${ }^{2) 13(18) 20(25) 27(31) 33)}$ Although numerous hypotheses ${ }^{2455)(899) 12(13) 29-31 / 38)}$ attempting to explain the formation of rICH including over-drainage of CSF have been suggested by case reports or literature reviews, none has been able to clearly demonstrate the associated definite underlying pathogenesis.

Though rICH is relatively rare complication, it may result in significant morbidity and mortality. ${ }^{3031)}$

Therefore, the immediate goal of this study was to evaluate and demonstrate the most approximate $\mathrm{rICH}$ pathogenesis by investigating the relationship between LD and POP rICH specially in the craniotomy for ruptured intracranial aneurysms.

\section{MATERIALS AND METHODS}

We retrospectively reviewed the medical records and radiographies of consecutive patients who underwent craniotomy for ruptured cerebral aneurysms at our institute from 1998 to 2004 . We excluded cases of unruptured cerebral aneurysms, ruptured aneurysms that were treated with both clipping and extraventricular drainage, endovascular embolization or operated using various skull base approaches including orbitozygomatic, lateral suboccipital, or different types of combined skull base approaches.

We identified age, sex, initial Hunt-Hess and Fischer grades, location of ruptured aneurysm, coagulation profiles, timing of surgery (early or delayed), presence or absence of hypertensive-hypervolemic $(\mathrm{H}-\mathrm{H})$ therapeutic intervention, history of hypertension, and LD and closed system negative pressure suction drain (SD) usage in each of the patients included in this study. $\mathrm{H}-\mathrm{H}$ therapy was conducted in compliance with an internationally recommended protocol. ${ }^{15)}$ Target levels of intracranial pressure, blood pressure (BP), and central venous pressure were also determined by the universal standards, ${ }^{16)}$ along with the POP transcranial Doppler result, and neurological signs and symptoms of the patient. POP rICH diagnosis was confirmed by using either the head computed tomography (CT) or magnetic resonance image (MRI). We then compared the incidence and characteristics of POP rICH between patients who had a perioperative $\mathrm{LD}$ and patients who did not, and analyzed variety of potential risk factors of $\mathrm{POP}$ rICH via univariate analysis and multivariate logistic regression analysis.

LD duration and the volume of CSF drain were also investigated in patients who had a perioperative LD. Final diagnosis of spontaneous SAH, ruptured cerebral aneurysm, microsurgical techniques, LD and SD management, and perioperative care were performed based on universal standards. The neurosurgeon conducting the procedure solely decided whether LD and suction drainage (SD) needed to be inserted or not, based on each patient's status including surgical field, initial radiographic findings and the surgeon's experience. During the study period, we exclusively used a lumbar kit from In- 
tegra-Neurosciences $^{\mathrm{TM}}$ (Plainsboro, NJ, USA) to drain of CSF, and Hemovac ${ }^{\circledR}$ (Zimmer Surgical, Inc., Dover, OH, USA) as a SD to drain epidural and/or subgaleal oozing blood and exudates.

For the statistical analysis, commercially available software, statistical package for the social science SPSS 19.0 (SPSS Inc., Chicago, IL, USA) was used. Demographic and clinical data with regard to the LD and $\mathrm{rICH}$ were compared using the t-test, $[\mathrm{chi}]^{2}$ test, ANOVA as appropriate. We also conducted multivariate logistic regression analyses upon possible clinical variables. All variables except age were considered as categorical. A $p$ value of $<0.05$ was considered as statistically significant.

\section{RESULTS}

Between 1998 and 2004, total 688 patients were deemed suitable based on inclusion criteria. Of these subjects, 80 patients had perioperative LD. The patients with LD have higher rate of SD placement compared to non-LD group and it was statistically significant (Table 1).

Table 1. Demographic and clinical characteristics of patients with an LD and without an LD

\begin{tabular}{|c|c|c|c|}
\hline Variables & $\operatorname{LD}(+)^{*}$ & $\operatorname{LD}(-)^{\dagger}$ & $p$ value \\
\hline No. of patients (\%) & $80(11.6)$ & $608(88.4)$ & \\
\hline Sex (Male:Female ratio) & $31: 49$ & $202: 406$ & 0.326 \\
\hline Age (years) & & & 0.057 \\
\hline Range & $26-67$ & $20-79$ & \\
\hline Mean & 49.88 & 52.52 & \\
\hline Hypertension history (\%) & $36(45.0)$ & $202(33.2)$ & 0.037 \\
\hline Hunt-Hess grade (\%) & & & 0.109 \\
\hline 1 & $3(3.7)$ & $52(8.5)$ & \\
\hline 2 & $40(50)$ & $339(55.8)$ & \\
\hline 3 & $17(21.3)$ & $116(19.1)$ & \\
\hline 4 & $15(18.7)$ & $87(14.3)$ & \\
\hline 5 & $5(6.3)$ & $14(2.3)$ & \\
\hline Fischer grade (\%) & & & 0.295 \\
\hline 1 & $1(1.2)$ & $4(0.7)$ & \\
\hline 2 & $10(12.5)$ & $123(20.2)$ & \\
\hline 3 & $38(47.5)$ & $290(47.7)$ & \\
\hline 4 & $31(38.8)$ & $191(31.4)$ & \\
\hline Location of aneurysms & & & 0.811 \\
\hline Anterior circulation & 69 & 524 & \\
\hline Posterior circulation & 1 & 14 & \\
\hline Multiple & 10 & 70 & \\
\hline Coagulopathy & 0 & 2 & 0.607 \\
\hline Surgery timing (\%) & & & 0.021 \\
\hline Early operation & $63(78.8)$ & $535(88.0)$ & \\
\hline Delayed operation & $17(21.2)$ & $73(12.0)$ & \\
\hline Insertion of SD (\%) & 77 (96.3) & $278(45.7)$ & 0.000 \\
\hline $\mathrm{H}-\mathrm{H}$ therapy (\%) & $60(75)$ & $408(67.1)$ & 0.155 \\
\hline
\end{tabular}

Values are presented as number (\%, if necessary)

* $\mathrm{LD}(+)$, Patients who underwent a perioperative lumbar drainage

${ }^{+} \mathrm{LD}(-)$, Patients who did not undergo a perioperative lumbar drainage

₹ Operation performed within 72 hours after rupture of cerebral aneurysm

$\mathrm{H}-\mathrm{H}$ therapy, Hypertensive-Hypervolemic therapy; SD, suction drainage 


\section{Incidence of POP rICH}

LD and POP rICH: Among 688 patients, 41 showed several kinds of POP hemorrhages on POP head CT. Of these patients, 17 had an LD and 24 did not. In the LD group (80 patients [Table 1]), 6 demonstrated tiny $\mathrm{ICH}$ in the operative field, and 1 had an intraventricular hemorrhage, and 10 presented POP rICH. Alternatively, in the non-LD group (608 patients [Table 1]), 8 had a minor amount (thinner than the skull table) of epidural hematoma (EDH) under the skull flap, 10 showed small amounts of ICH in the operative site, 1 presented thin $\mathrm{EDH}$ on opposite side of craniotomy, and 5 demonstrated POP rICH. All POP EDHs and operative site POP ICHs were not amenable to surgical removal. POP $\mathrm{rICH}$ incidence was $12.5 \%$ in the $\mathrm{LD}$ and $0.8 \%$ in the non- $\mathrm{LD}$ group. Additionally, there was a statistically significant difference in POP rICH incidence between these two groups $(p=0.000)$ (Table 2$)$.
SD and POP rICH: From the total 688 patients, 355 had an SD. Of these 355, 15 demonstrated POP rICH. Namely, all $15 \mathrm{rICH}$ patients in this study had an SD. Subsequently, the rate of $\mathrm{rICH}$ was $4.2 \%(15 / 355)$ in patients with an SD (Table 2).

Simultaneous insertion of an LD and an SD, and POP rICH: Among the entire 688 patients, 77 were treated by using an LD and an SD simultaneously (Table 1). Of these 77 patients, POP rICH developed in 10, then the resulting POP $\mathrm{rICH}$ rate was $13.0 \%(10 / 77)$. There was statistically significant difference in of POP rICH incidence between simultaneous use of $\mathrm{LD}$ and SD group (10/77, 13\%) and non-simultaneous group (5/611, $0.008 \%)(p=0.000)$ (Table 1, 2).

Amount of CSF drainage via LD and POP rICH: Mean drained CSF through LD per day was below 50 $\mathrm{mL} /$ day in 41 patients, between $50-100 \mathrm{~mL}$ /day in 23 , and above $100 \mathrm{~mL} /$ day in 16 . The POP rICHs were de-

Table 2. Comparison of clinical characteristics of POP rICH between patients with and without an LD

\begin{tabular}{|c|c|c|c|}
\hline Variables & $\operatorname{LD}(+)^{\star}(n=80)$ & $\operatorname{LD}(-)^{\dagger}(n=608)$ & $p$ value \\
\hline No. of patients with rlCH (\%) & $10(12.5)$ & $5(0.8)$ & 0.000 \\
\hline Location of $\mathrm{rlCH}$ & & & 0.591 \\
\hline Cerebellum & 9 & 4 & \\
\hline Contralateral to operative site & 3 & 1 & \\
\hline Ipsilateral to operative site & 4 & 1 & \\
\hline Bilateral hemispheres & 2 & 2 & \\
\hline Cerebral hemisphere & 1 & 1 & \\
\hline Contralateral to operative site & 1 & 1 & \\
\hline Treatment & & & 0.143 \\
\hline Conservative & 10 & 4 & \\
\hline Surgical & 0 & 1 & \\
\hline Outcome & & & 0.648 \\
\hline No residual neurologic deficits & 2 & 1 & \\
\hline Temporary neurologic deficits & 4 & 1 & \\
\hline Permanent neurologic deficits & 1 & 0 & \\
\hline Death & 3 & 3 & \\
\hline Suction drainage & & & 0.000 \\
\hline Yes & 10 & 5 & \\
\hline No & 0 & 0 & \\
\hline
\end{tabular}

Values are presented as number (\%) if necessary

* $\mathrm{LD}(+)$, Patients who underwent a perioperative lumbar drainage

${ }^{+} \mathrm{LD}(-)$, Patients who did not undergo a perioperative lumbar drainage

$\mathrm{POP}$, Postoperative; $\mathrm{rlCH}$, remote intracerebral hemorrhage 
veloped in 4 patients of $<50 \mathrm{~mL}$ group (9.8\%), 6 of 50 $100 \mathrm{~mL}(26.1 \%)$, and none from the $>100 \mathrm{~mL}(0 \%)$, in each (Table 3).

Duration of LD and POP rICH: Number of patients that showed POP rICH in relation to LD duration are summarized in Table 3. There was significant difference in $\mathrm{rICH}$ frequency in association with the duration of LD $(p=0.026)$ (Table 3).

Prior history of hypertension and POP rICH: Among 688 patients, 238 had a medical history of hypertension and 450 did not (Table 1). Considering 238, 5 patients developed POP rICH and of the remaining 450, 10 patients resulted in POP rICH. Then, the incidences of

Table 3. Clinical features of patients who had an LD and developed $\mathrm{rICH}$

\begin{tabular}{|c|c|c|}
\hline Features & $\begin{array}{l}\text { POP rICH (\%) } \\
\quad(n=10)\end{array}$ & $p$ value \\
\hline Amount of drained CSF (\%) & & 0.040 \\
\hline$<50$ mL/day $(\mathrm{n}=41)$ & $4(9.8)$ & \\
\hline 50-100 mL/day $(n=23)$ & $6(26.1)$ & \\
\hline$>100$ mL/day $(\mathrm{n}=16)$ & $0(0)$ & \\
\hline Duration of LD (\%) & & 0.026 \\
\hline Only during surgery $(\mathrm{n}=7)$ & $3(42.9)$ & \\
\hline 1 day $(n=38)$ & $3(7.9)$ & \\
\hline 2 days $(n=4)$ & $2(50)$ & \\
\hline 3 days $(n=6)$ & $0(0)$ & \\
\hline 4 days $(n=5)$ & $0(0)$ & \\
\hline 5 days $(n=6)$ & $0(0)$ & \\
\hline 6 days $(n=5)$ & $2(40)$ & \\
\hline 7 days $(n=5)$ & $0(0)$ & \\
\hline 8 days $(n=1)$ & $0(0)$ & \\
\hline 9 days $(n=3)$ & $0(0)$ & \\
\hline Hypertension history & & 0.089 \\
\hline Yes & 2 & \\
\hline No & 8 & \\
\hline Lumbar and suction drainage & & 0.000 \\
\hline Yes & 10 & \\
\hline No & 0 & \\
\hline Hypertensive-Hypervolemic* & & 0.089 \\
\hline Yes & 8 & \\
\hline No & 2 & \\
\hline
\end{tabular}

Values are presented as number (\%, if necessary)

* Hypertensive-Hypervolemic, Hypertensive-Hypervolemic therapy

LD, lumbar drainage; POP, postoperative; $\mathrm{rlCH}$, remote intracerebral hemorrhage; CSF, cerebrospinal fluid
rICH were $2.1 \%$ and $2.2 \%$, respectively (Table 1,4 ).

$\mathrm{H}-\mathrm{H}$ therapy and POP rICH: Among the included 688 patients, 468 underwent prophylactic or therapeutic $\mathrm{H}-\mathrm{H}$ therapy, irrespective of its duration. Among these 468 patients, 13 showed POP rICH. The rates of $\mathrm{rICH}$ were $2.8 \%$ and $0.9 \%$ in the group of patients who had $\mathrm{H}-\mathrm{H}$ therapy and who did not, respectively (Table 1,4 ).

Multivariate analysis: Higher volume of the subarachnoid blood clot (or Fisher grade) indicated an equally greater risk of developing POP $\mathrm{rICH}$, and this correlation was statistically significant $(p=0.046)$ (Table 4$)$. However, the Fisher grade was not an independent risk factor in the multivariate logistic regression analysis (Table 5). LD was the only independent risk factor associated with the formation of POP rICH in the multivariate analysis. Additionally, SD also was not an independent risk factor (Table 5).

\section{Location of POP rICH}

In a group of 80 patients who underwent LD, 9 exhibited POP rICH in the cerebellum (3, ipsilateral to the operative site; 4 , contralateral; 2 , bilateral) and 1 developed POP rICH at basal ganglia contralateral to the operative side (Table 2). In the group that did not undergo LD, 4 of 608 patients developed POP rICH in the cerebellum (1, ipsilateral to the craniotomy; 1 , contralateral; 2 , bilateral) and 1 presented POP rICH at the contralateral basal ganglia (Table 2). Furthermore, the location of POP $\mathrm{rICH}$ was neither consistent nor statistically significant with regard to the use of LD ( $p=0.591)$ (Table 2).

\section{Management and outcome of POP rICH}

Table 2 demonstrates POP rICH management methods and their results in 10 of 80 patients who had LD and in 5 out of 608 patients who did not. The temporary neurological deficits were dizziness, sense of imbalance, hemiparesis, tremor, and confusion. And the permanent neurological deficit was hemiparesis. The overall mortality associated with POP rICH was $40.0 \%(6 / 15)$ in this study (Table 2). 
Table 4. Evaluation of risk factors for postoperative rICH development in this study

\begin{tabular}{|c|c|c|c|}
\hline Variables & $\mathrm{rlCH}(+)^{*}$ & $\mathrm{rICH}(-)^{\dagger}$ & $p$ value \\
\hline No. of patients (\%) & 15 & 673 & \\
\hline Sex (Male : Female ratio) & $8: 7$ & $225: 448$ & 0.107 \\
\hline Age (years) & & & 0.433 \\
\hline Range & $28-70$ & $20-79$ & \\
\hline Mean & 49.87 & 52.27 & \\
\hline Hypertension history (\%) & & & 0.057 \\
\hline Yes & 5 & 233 & \\
\hline No & 10 & 440 & \\
\hline Hunt-Hess grade (\%) & & & 0.851 \\
\hline \multicolumn{4}{|l|}{0} \\
\hline 1 & 1 & 54 & \\
\hline 2 & 7 & 372 & \\
\hline 3 & 3 & 130 & \\
\hline 4 & 3 & 99 & \\
\hline 5 & 1 & 18 & \\
\hline Fischer grade (\%) & & & 0.046 \\
\hline 1 & 1 & 4 & \\
\hline 2 & 2 & 131 & \\
\hline 3 & 8 & 320 & \\
\hline 4 & 4 & 218 & \\
\hline Coagulopathy & 0 & 2 & 0.833 \\
\hline Surgery timing (\%) & & & 0.977 \\
\hline Early operation $^{\ddagger}$ & 13 & 585 & \\
\hline Delayed operation & 2 & 88 & \\
\hline $\mathrm{H}-\mathrm{H}$ therapy (\%) & & & 0.089 \\
\hline Yes & 13 & 455 & \\
\hline No & 2 & 218 & \\
\hline
\end{tabular}

Values are presented as number

* $\mathrm{rlCH}(+)$, patients who developed remote intracerebral hemorrhage

${ }^{+} \mathrm{rlCH}(-)$, patients who did not develop remote intracerebral hemorrhage

‡ Early Operation, Operation performed within 72 hours after rupture of cerebral aneurysm

$\mathrm{H}-\mathrm{H}$, Hypertensive-Hypervolemic

Table 5. Multivariate analysis for the clinical features of remote intracerebral hemorrhage

\begin{tabular}{llll}
\hline Variables & OR & 95\% Cl & $p$ value \\
\hline Sex (Female/Male) & 0.475 & $0.148-1.525$ & 0.211 \\
Age (years) & 0.998 & $0.958-1.038$ & 0.905 \\
Hunt-Hess Grade & 1.183 & $0.623-2.246$ & 0.607 \\
Fischer Grade & 0.477 & $0.215-1.059$ & 0.248 \\
Surgery timing (Early*/ Delayed) & 2.083 & $0.408-10.622$ & 0.377 \\
Hypertension history (No/Yes) & 1.208 & $0.410-3.559$ & 0.732 \\
Suction drainage insertion (No/Yes) & 0.000 & 0.000 & 0.994 \\
Lumbar drainage insertion (No/Yes) & 0.112 & $0.035-0.356$ & 0.0002 \\
H-H therapy (No/Yes) & 0.344 & $0.074-1.607$ & 0.175 \\
\hline
\end{tabular}

* Early, Operation performed within 72 hours after rupture of cerebral aneurysm

$\mathrm{H}-\mathrm{H}$, Hypertensive-Hypervolemic; OR, odd ratio; $\mathrm{Cl}$, confidence interval 


\section{DISCUSSION}

Although LD has frequently been used in a variety of conditions in neurosurgery, it has been clearly understood that the over-drainage of CSF irrespective of the routes, may induce a plethora of complications ${ }^{4)}$ 8)1421212428830)31) with the rICH being considered the most critical POP event. Fortunately, it has been rare adverse event. ${ }^{12303131)}$ Numerous authors and researchers have described the incidence and suggested the pathogenesis of POP rICH in their studies. ${ }^{58-10) 13118(20) 222(25) 27(29) 33(35) 36) 38)}$ During their descriptions, several causative factors have been proposed, however, due to the low incidence of $\mathrm{rICH}$ and the numerous limitations of their reports, the definite pathogenesis and related risk factors associated with POP rICH remain unclear. Subsequently, we aimed to identify if the CSF loss via LD influenced the occurrence of POP rICH, and other risk factors that contributed to the development of POP rICH if any.

\section{Incidence of POP rICH}

Although the characteristics of two groups (LD group and non-LD group) in this study were significantly different in the frequency of history of hypertension, timing of surgery and placement of SD, these variables were not independent risk factors in the multivariate analysis (Table 5). Then, we would decide to directly compare the LD group to the non-LD group. Subsequently, POP $\mathrm{rICH}$ incidence was significantly higher in the LD group $(12.5 \%)$ than non-LD group $(0.8 \%) \quad(p=0.000)$ (Table 2). And the overall incidence of POP rICH was $2.2 \%$ (Table 2).

In a review of 4992 intracranial procedures, Kalfas and Little identified that rICH accounted for 18\% (7/40) of all POP intracranial hemorrhages. ${ }^{12)}$ Their POP rICH rate was approximately $0.1 \%$. Waga et al. ${ }^{35)}$ in 1983 reported 4 cases of POP rICH after neurosurgical procedures such as microsurgical repair of ruptured cerebral aneurysm in patients with recent SAH. However, they did not specify rICH incidence. In 2001, Friedman et al. ${ }^{10)}$ published that POP rICH occurred in approximately $0.6 \%$ of all supratentorial craniotomies and $2.8 \%$ of all craniotomies to treat unruptured cerebral aneurysm. In 1996, Lee et al. ${ }^{20)}$ reported that the incidence of POP $\mathrm{rICH}$ in 720 ruptured intracranial aneurysms was below $1 \%$, which was lower than our result (2.2\%). They claimed that the sudden elevation of $\mathrm{BP}^{10) 12(35)}$ during the remission period of the cerebral vasospasm was the root cause of the rICH.

POP rICH incidence (2.2\%) according to our study is relatively lower than that after direct surgical repair of ruptured and/or unruptured cerebral aneurysms as reported by others. ${ }^{2) 10}$ It is possible that this minor difference may provide an extremely important clue, or at least an incomplete clue, regarding POP rICH pathogenesis. It suggested that although CSF loss was exactly the same amount and/or velocity both during surgery and POP period, the displacement or shrinkage of cerebrum and cerebellum was less in patients with recent SAH than in those with unruptured aneurysm without $\mathrm{SAH}$ due to the presence of an irritated and edematous cerebrum and cerebellum by the subarachnoid blood.

\section{Location of POP rICH}

Studies have previously demonstrated that certain cases of POP rICH following intracranial aneurysm surgery occurred in the cerebral hemisphere contralateral to the operative site, while other reports suggested that POP rICH typically developed in the contralateral cerebellar hemisphere. ${ }^{2) 182031)}$ In 2001, Friedman et al. ${ }^{10)}$ suggested that the typical location of POP rICH was the superior cerebellar folia. A systematic review of literatures on the remote cerebellar hemorrhage after supratentorial procedures, was published by Sturiale et al. ${ }^{31)}$ in 2016, reported that even though their report was confined to the cerebellar hemorrhage, they noted that $55.1 \%$ of cerebellar rICH were bilateral, and $21.9 \%$ were contralateral to the supratentorial surgery. In our study, 13 out of 15 patients with POP rICH appeared in the cerebellum (Table 2). Then, we assumed that although POP rICH characteristically occurred more often in the cerebellum than in the cerebrum, it did not have any such preference in the laterality. 


\section{Pathogenesis of POP rICH}

As described before, the overall POP rICH incidence was $2.2 \%(15 / 688)$ in our study and among these POP rICH cases, the incidences were $12.5 \%(10 / 80)$ and $0.8 \%$ $(5 / 608)$ in the patients who had LD and who did not, respectively (Table 2). This result implicated that the loss of CSF through LD might be a crucial causative factor associated with the generation of POP rICH. Moreover, among our study population of 688,77 patients had an LD and an SD simultaneously. Of these 77, 10 patients presented POP rICH. Subsequently, POP rICH incidence for this group (LD+SD) was 13.0\%. Numerically, simultaneous application of an LD and an SD was a more impactive risk factor associated with the formation of POP rICH than using LD or SD alone in this study (Table 1,2). Typically, the drained liquid material through SD was composed of blood components and CSF. However, it was not possible to isolate CSF from blood components in the clinical practice. Accordingly, we could not quantify the drained CSF via SD. We hypothesized that more rapid and/or larger amount of CSF loss per given time period through LD and SD might happen in this group $(\mathrm{LD}+\mathrm{SD})$. If it was so, it might have had a considerable impact on the occurrence of POP rICH. This observation might prove to be critical as conclusion of present study.

Although the initial Fisher grade of patient was not an independent risk factor associated with the formation of POP rICH in the multivariate analysis, we noted that the initial Fisher grade demonstrated a statistically significant correlation with the risk of developing POP rICH in the univariate analysis (Table 4, 5). We presumed the background of this result was that the degree of dysfunction in brain autoregulation at least partially, depends on the volume of subarachnoid blood clot, and this dysfunction might partially at least, generate POP rICH.

Unexpectedly, the authors failed to verify that POP $\mathrm{rICH}$ incidence was directly proportional to the amount and/or duration of CSF drain via LD in spite of these two parameters (amount and duration of CSF drain via LD) showed statistically significant correlations in the frequency of POP rICH (Table 3). It was our under- standing that this failure was due to the small number of patients involved in this study and the inherent limitations of our retrospective study. We believe that CSF drain velocity per defined unit time via $\mathrm{LD}$ or any route has a greater impact on the development of POP rICH, rather than the total amount and/or duration of CSF drain. Furthermore, the amount and/or duration of CSF drain without POP rICH formation might vary greatly among patients. A large scale, well designed multicenter prospective research effort on this subject should be created in the future.

According to relatively early period of case reports on the POP rICH, fluctuation or sudden elevation of $\mathrm{BP}$ during the craniotomy, initial POP period, vasospasm, $\mathrm{H}-\mathrm{H}$ therapy, autoregulatory impairment, and perioperative coagulopathy might potentially cause POP

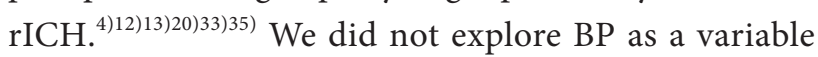
associated with the genesis of POP $\mathrm{rICH}$ since medical records showed that no fluctuation or sudden elevation of BP during the above mentioned intervals or periods despite our meticulous examination of the records of the entire 688 included patients. Here, 2 patients without LD proved to have coagulopathy although they did not present POP rICH. Then, even though the number of coagulopathy cases in present study was too insignificant to reach a certain opinion, we believe that after thoroughly examining relevant literatures along with our collective experiences, association between coagulopathy and POP rICH was not evident despite of a few literatures contradicting the same issue. ${ }^{4-6) 10) 12(18) 31333}$

Alternatively, the consensus that the position of the patient's head during craniotomy with microsurgery has a certain degree of influence in the development of POP rICH is relatively vague. Compression of internal jugular vein at the transverse process of the first cervical vertebra and resulting intracranial venous hypertension as a consequence of the patient's head position during the craniotomy may cause POP $\mathrm{rICH} .{ }^{29)}$ Although our standard protocol suggest keeping the head position as neutral as possible during the frontotemporal craniotomy, we could not absolutely exclude the chance of surgical head position of patient as an underlying cause of the 


\section{POP rICH.}

Brain sagging as a basic etiology of POP rICH, was introduced following intraoperative and/or perioperative CSF over- drain in craniotomy. ${ }^{421)}$ It might be possible that the cerebellar displacement and sagging ${ }^{41922126)}$ due to the over-loss of CSF leads to transient stretching and angulation of superiorly directed cerebellar veins draining towards the cephalad direction into the deep venous system. Finally, occlusion of these cerebellar veins sufficiently produces cerebellar rICH and/or venous infarction. ${ }^{4) 2122)}$

Recently, a few authors ${ }^{34)}$ coined and used a new term that intrinsically and potentially had identical pathogenesis as POP rICH, but showed different clinical phenomena from rICH, the POP 'pseudohypoxic brain swelling' that presented as consciousness disturbance, brain stem dysfunction, and generalized seizures after an uneventful craniotomy. These authors proposed a common pathogenesis that the diffuse brain swelling, particularly swelling of the deep brain nuclei such as basal ganglia and thalami on head CT or MRI, was ascribed to the over-drain of CSF through the SD. After their introduction of a new concept, a few neurosurgeons have reported such adverse events. ${ }^{1132(37)}$ In 2019, Katsevman et al. ${ }^{17)}$ experienced a PRES in a patient and reviewed the related literatures on the PRES.7) They admitted CSF over-drainage (CSF hypovolemia) could be one of the several possible causative pathophysiologic mechanisms associated with the occurrence of PRES. If it is so, it is possible that the pseudohypoxic brain swelling and PRES may have a common etiological factor.

Given the situation we do not have choice but to accept that the unidentified or unknown POP clinical settings including neurosurgical conditions, which can violently disturb homeostasis or normal internal milieu of the brain may occur beyond the known clinical conditions as specified in Fig. 1 and 2, because of the CSF overloss. Upon further consideration, we understood that the basic and core pathogeneses of POP rICH, brain sag, pseudohypoxic brain swelling and some of PRES might

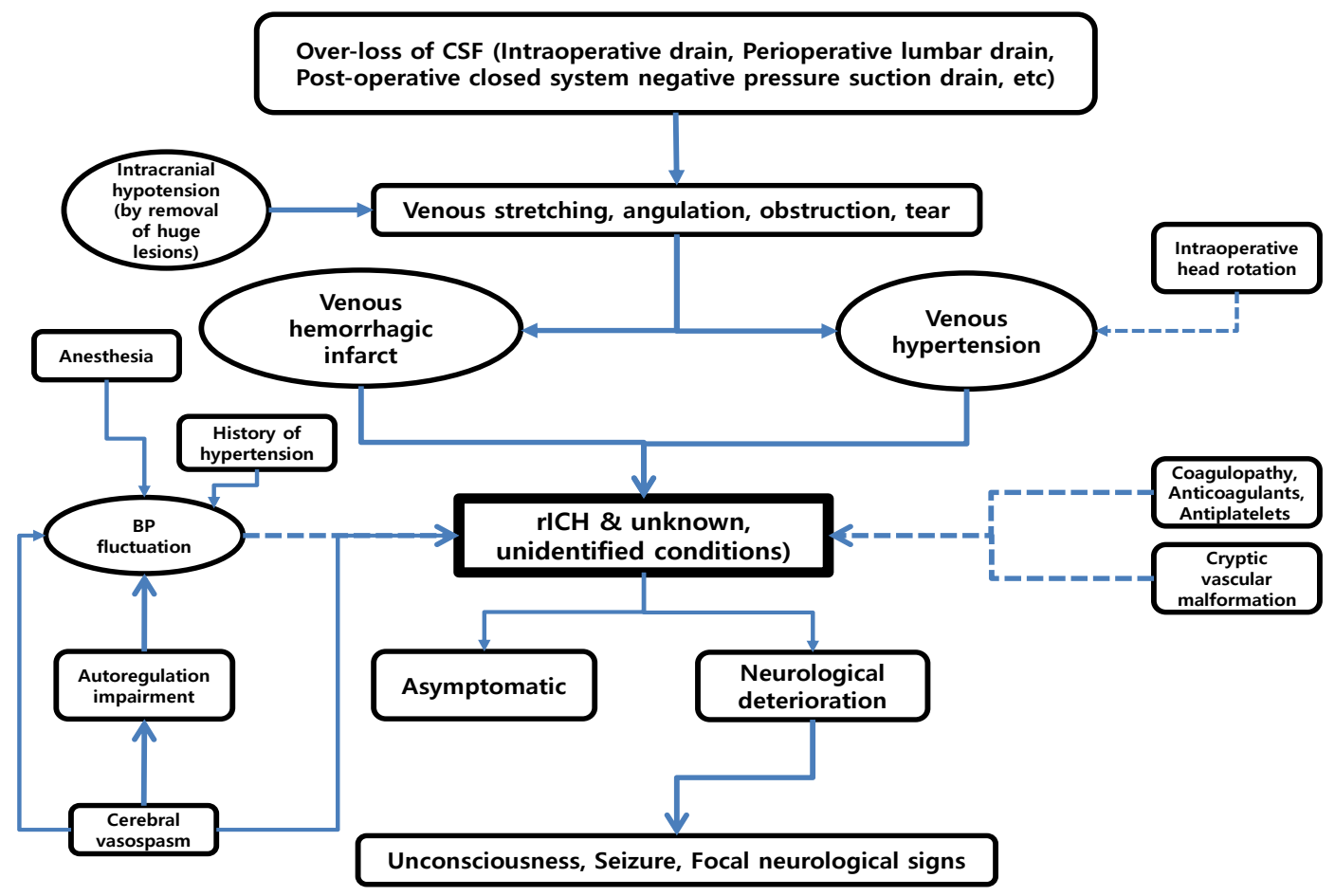

Fig. 1. Here, we demonstrate and summarize potential pathogenesis and progress associated with the development of riCH and unknown, unidentified neurological conditions. The arrows and lines of dotted, and the arrow and relatively thinner line indicate low and moderate levels of possible pathogeneses, respectively. BP, blood pressure; CSF, cerebrospinal fluid; LD, lumbar drainage; SD, suction drainage. rICH, remote intracerebral hemorrhage. 


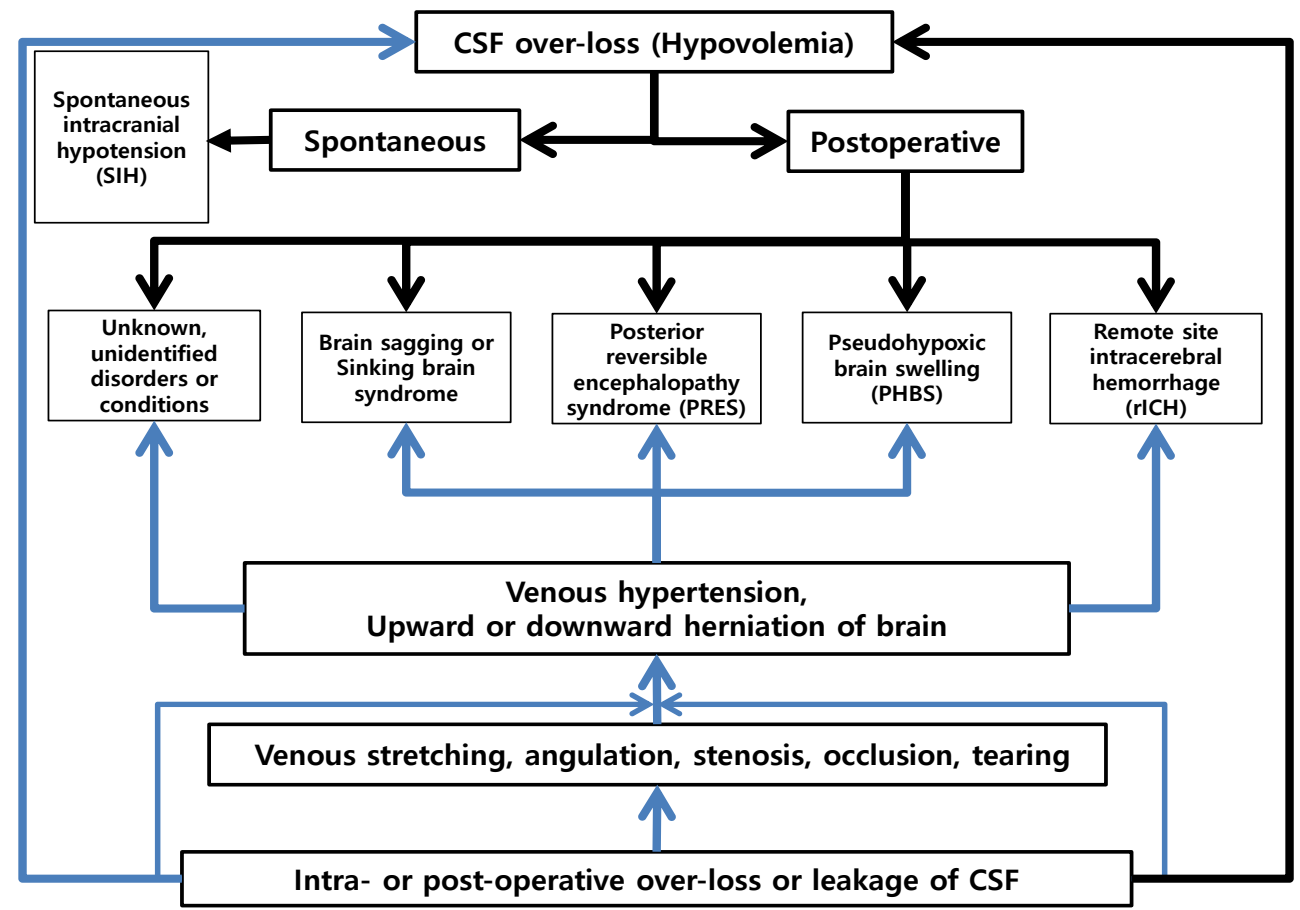

Fig. 2. Suggested pathogenesis of postoperative (POP) remote intracerebral hemorrhage $(\mathrm{rlCH})$ and other feasible neurological disorders or phenomena. CSF over-loss (hypovolemia) is the initial event and/or root cause which may trigger diverse neurological phenomena or complications including POP rICH. CSF, cerebrospinal fluid.

be essentially identical (Fig. 1, 2). ${ }^{26)}$ Additionally according to the authors' review of the literatures, although a number of potential etiologies might be possible, ${ }^{1231)}$ the most impactfully, frequently, and continuously suggested key point, was the reduction in intracranial CSF volume (CSF hypovolemia). ${ }^{2448-10) 25(31) 38 \text { ) }}$

Comprehensively, we propose the pathogenesis of POP rICH considering the results of this study and a thorough review of the literatures is shown in Fig. 1 and 2. However, the POP rICH pathogenesis may be more complex than expected.

\section{Limitations of this study}

This study has numerous weaknesses and there may be more drawbacks apart from those mentioned below. First, there are several inherent limitations due to the retrospective natures of this study, including an absence of a corresponding control group, missing data that was neither documented nor checked up. Second, we did not subdivide the volume of $\mathrm{rICH}$ in the POP rICH patients and the corresponding volume of CSF loss through the LD.
However, this two might be associated with each other. Third, we considered and analyzed CSF drain for only the 24 hours ( 1 day) basis without checking the velocity of CSF drain via LD within more segmented time periods. Fourth, we did not subdivide the patients into age groups which might have an effect on the frequency, appearance, and volume of POP rICH. Finally, we did not consider CSF drain volume via SD, although it was not an independent risk factor associated with the emergence of POP rICH in the multivariate analysis.

\section{CONCLUSIONS}

The LD is characteristically used for a variety of neurosurgical procedures including cerebral aneurysm. However, it should be used in only specific and selected conditions, and rapid and/or large amount of CSF drain through LD should be prevented and closely monitored. Additionally, while treating patients with any CSF drain routes, neurosurgeons should be aware of the possibility 
of POP rICH even after a smooth, uneventful, and successful procedure.

\section{ACKNOWLEDGEMENTS}

The authors would like to thank Prof. DP Jang (Hanyang University, Korea) for the contribution of statistical analysis and its interpretation.

\section{Disclosure}

The authors report no conflict of interest concerning the materials or methods used in this study or the findings specified in this paper.

\section{REFERENCES}

1. Atkinson JL, Weinshenker BG, Miller GM, Piepgras DG, Mokri B. Acquired Chiari I malformation secondary to spontaneous spinal cerebrospinal fluid leakage and chronic intracranial hypotension syndrome in seven cases. J Neurosurg. 1998 Feb;88(2):237-42.

2. Baek IH, Park KY, Lee JW, Huh SK. Remote cerebellar hemorrhage after surgery for an unruptured aneurysm. J Cerebrovasc Endovasc Neruosurg. 2008 Sep;10(3):454-8.

3. Basauri LT, Concha-Julio E, Selman JM, Cubillos P, Rufs J. Cerebrospinal fluid spinal lumbar drainage: indications, technical tips, and pitfalls. Crit Rev Neurosurg. 1999 Jan;9(1):21-7.

4. Borkar SA, Lakshmiprasad G, Sharma BS, Mahapatra AK. Remote site intracranial haemorrhage: a clinical series of five patients with review of literature. Br J Neurosurg. 2013 Dec;27(6):735-8.

5. Brisman MH, Bederson JB, Sen CN, Germano IM, Moore F, Post KD. Intracerebral hemorrhage occurring remote from the craniotomy site. Neurosurgery. 1996 Dec;39(6):1114-1121; discussion 1121-2.

6. Chang SH, Yang SH, Son BC, Lee SW. Cerebellar hemorrhage after burr hole drainage of supratentorial chronic subdural hematoma. J Korean Neurosurg Soc. 2009 Dec;46(6):592-5.

7. Delgado-López PD, Garcés-Pérez G, García-Carrasco J, Alonso-García E, Gómez-Menéndez AI, Martín-Alonso J. Posterior reversible encephalopathy syndrome with status epilepticus following surgery for lumbar stenosis and spondylolisthesis. World Neurosurg. 2018 Aug;116:309-15.
8. Doddamani RS, Sawarkar D, Meena RK, Gurjar H, Singh PK, Singh M, et al. Remote cerebellar hemorrhage following surgery for supratentorial lesions. World Neurosurg. 2019 Jun;126:e351-9.

9. Friedman JA, Ecker RD, Piepgras DG, Duke DA. Cerebellar hemorrhage after spinal surgery : report of two cases and literature review. Neurosurgery. 2002 Jun;50(6):1361-3; discussion 1363-4.

10. Friedman JA, Piepgras DG, Duke DA, McClelland RL, Bechtle PS, Maher CO, et al. Remote cerebellar hemorrhage after supratentorial surgery. Neurosurgery. 2001 Dec;49(6):1327-40.

11. Honeybul S. Sudden death after cranioplasty. J Korean Neurosurg Soc. 2016 Mar;59(2):182-4.

12. Kalfas IH, Little JR. Postoperative hemorrhage: a survey of 4992 intracranial procedures. Neurosurgery. 1988 Sep;23(3):343-7.

13. Kang YG, Chung H, Lee SP, Choi KH, Yeo HT, Rhee JK. Remote intracerebral hematoma after supratentorial craniotomy. J Korean Neurosurg Soc. 1996 Sep;25(9):1910-6.

14. Kasuya H, Shimizu T, Kagawa M. The effect of continuous drainage of cerebrospinal fluid in patients with subarachnoid hemorrhage : a retrospective analysis of 108 patients. Neurosurgery. 1991 Jan;28(1):56-9.

15. Kassell NF, Sasaki T, Colohan ART, Nazar G. Cerebral vasospasm following aneurysmal subarachnoid hemorrhage. Stroke. 1985 Jul-Aug;16(4):562-72.

16. Kassell NF, Torner JC, Haley Jr EC, Jane JA, Adams HP, Kongable GL, et al. The international cooperative study on the timing of aneurysm surgery. Part 1: Overall management results. J Neurosurg. 1990 Jul;73(1):18-36.

17. Katsevman GA, Turner RC, Cheyuo C, Rosen CL, Smith MS. Post-partum posterior reversible encephalopathy syndrome requiring decompressive craniectomy: case report and review of the literature. Acta Neurochir (Wien). 2019 Feb;161(2):217-24.

18. Kim SH, Lee HK, Moon JK Kim CH, Choi JH. Remote cerebellar hemorrhage after supratentorial aneurysm surgery; report of 2 cases. J Cerebrovasc Endovasc Neruosurg. 2008 Dec;10(4):570-4.

19. Komotar RJ, Ransom ER, Mocco J, Zacharia BE, McKhann GM 2nd, Mayer SA, et al. Critical postcraniotomy cerebrospinal fluid hypovolemia : risk factors and outcome analysis. Neurosurgery. 2006 Aug;59(2):284-90.

20. Lee JW, Yim MB, Lee JC, Son EI, Kim DW, Kim IH. Intracerebral hemorrhage remote from the site of aneurysm surgery. J Korean Neurosurg Soc. 1996 Apr;25(4):834-41.

21. Liu JKC. Neurologic deterioration due to brain sag after bilateral craniotomy for subdural hematoma evacuation. World Neurosurg. 2018 Jun;114:90-3. 
22. Mandonnet E, Faivre B, Bresson D, Cornelius J, Guichard JP, Houdart E, et al. Supratentorial craniotomy complicated by an homolateral remote cerebellar hemorrhage and a controlateral perisylvian infarction : case report. Acta Neurochir (Wien). 2010 Jan;152(1):169-72.

23. McDonald JV, Klump TE. Intraspinal epidermoid tumors caused by lumbar puncture. Arch Neurol. 1986 Sep;43(9):936-9.

24. Motoyama Y, Nakajima T, Takamura Y, Nakazawa T, Wajima D, Takeshima Y, et al. Risk of brain herniation after craniotomy with lumbar spinal drainage: a propensity score analysis. J Neurosurg. 2018 Jun:1-11.

25. Papanastassiou V, Kerr R, Adams C. Contralateral cerebellar hemorrhagic infarction after pterional craniotomy: report of five cases and review of the literature. Neurosurgery. 1996 Oct;39(4):841-51; discussion 851-2.

26. Park CW. Postoperative cerebrospinal fluid hypovolemia in neurosurgery. J Neurointensive Care. 2018;1(1):3-6.

27. Park SJ Lee MK, Kim DJ. Supratentorial surgery complicated by cerebellar hemorrhage: report of three cases. J Korean Neurosurg Soc. 1997 Jul;26(7):1011-6.

28. Samadani U, Huang JH, Baranov D, Zager EL, Grady MS. Intracranial hypotension after intraoperative lumbar cerebrospinal fluid drainage. Neurosurgery. 2003 Jan;52(1):148-51; discussion 151-2.

29. Seoane E, Rhoton AL Jr. Compression of the internal jugular vein by the transverse process of the atlas as the cause of cerebellar hemorrhage after supratentorial craniotomy. Surg Neurol. 1999 May;51(5):500-5.

30. Sturiale CL, Rossetto M, Ermani M, Baro V, Volpin F, Milanese L, et al. Remote cerebellar hemorrhage after spinal pro- cedures (part 2): a systematic review. Neurosurg Rev. 2016 Jul;39(3):369-76.

31. Sturiale CL, Rossetto M, Ermani M, Volpin F, Baro V, Milanese L, et al. Remote cerebellar hemorrhage after supratentorial procedures (part 1): a systematic review. Neurosurg Rev. 2016 Oct;39(4):565-73.

32. Sviri GE. Massive cerebral swelling immediately after cranioplasty, a fatal and unpredictable complication: report of 4 cases. J Neurosurg. 2015 Nov;123(5):1188-93.

33. van Calenbergh F, Goffin J, Plets C. Cerebellar hemorrhage complicating supratentorial craniotomy: report of two cases. Surg Neurol. 1993 Oct;40(4):336-8.

34. van Roost D, Thees C, Brenke C, Oppel F, Winkler PA, Schramm J. Pseudohypoxic brain swelling : a newly defined complication after uneventful brain surgery, probably related to suction drainage. Neurosurgery. 2003 Dec;53(6):1315-26; discussion 1326-7.

35. Waga S, Shimosaka S, Sakakura M. Intracerebral hemorrhage remote from the site of the initial neurosurgical procedure. Neurosurgery. 1983 Dec;13(6):662-5.

36. Yasargil MG, Yonekawa Y. Results of microsurgical extra-intracranial arterial bypass in the treatment of cerebral ischemia. Neurosurgery. 1977 Jul-Aug;1(1):22-4.

37. Yokota H, Yokoyama K, Miyamoto K, Nishioka T. Pseudohypoxic brain swelling after elective clipping of an unruptured anterior communicating artery aneurysm. Clin Neurol Neurosurg. 2009 Dec;111(10):900-3.

38. You SH, Son KR, Lee NJ, Suh JK. Remote cerebral and cerebellar hemorrhage after massive cerebrospinal fluid leakage. J Korean Neurosurg Soc. 2012 Apr;51(4):240-3. 